Gemeinschaftsaufgabe Regionale Daseinsvorsorge

\section{Kein Königsweg für die Kommunen}

Die Bundestagsfraktion Bündnis 90/Die Grünen hat gefordert, dass zusätzlich zu den bereits vorhandenen Gemeinschaftsaufgaben von Bund und Ländern nach Art. 91a GG eine neue Gemeinschaftsaufgabe „Regionale Daseinsvorsorge" (GRD) eingeführt werden sollte. Auch in der SPD gibt es entsprechende Vorschläge. Dahinter steht der Befund, dass es mit den bisherigen Maßnahmen - nicht zuletzt mit den vorhandenen Gemeinschaftsaufgaben „Verbesserung der regionalen Wirtschaftsstruktur" sowie „Verbesserung der Agrarstruktur und des Küstenschutzes" - nicht gelungen ist, die regionalen Disparitäten in Deutschland nachhaltig zu reduzieren. Trotz positiver Entwicklungen in einzelnen Landesteilen sind viele strukturschwache Regionen bei ihrer Wirtschaftsleistung sowie ihrer Ausstattung mit Einrichtungen der Daseinsvorsorge deutlich hinter den prosperierenden Regionen zurückgeblieben und von Abwanderung betroffen. In der Bevölkerung der strukturschwachen Regionen hat sich das Empfinden verbreitet, „abgehängt“ zu sein und immer weniger Einfluss auf die regionale Entwicklung nehmen zu können. Infolge dieser Tendenzen hat sich die Politik in den vergangenen Jahren verstärkt mit der Frage beschäftigt, wie für mehr „Gleichwertigkeit der Lebensverhältnisse" zwischen den Regionen gesorgt werden kann, speziell hinsichtlich der Daseinsvorsorge.

Mit Daseinsvorsorge ist ein bunter Strauß von Einrichtungen gemeint, die einen lokalen oder regionalen Nutzerkreis haben und zumeist zum Aufgabenbereich der Kommunen zählen, z.B. Wasserversorgung, Schulen, Feuerschutz, Kultureinrichtungen. Auch private Dienstleistungen wie Arztpraxen, Einzelhandelsgeschäfte oder Gaststätten, deren örtliche Verfügbarkeit für private Haushalte von großer Bedeutung ist, werden zur Daseinsvorsorge gezählt. Ein Mangel an derartigen Einrichtungen wirkt sich auch negativ auf die wirtschaftliche Entwicklung aus, insbesondere auf die regionale Versorgung mit Fachkräften. Bislang liegt es in erster Linie im Ermessen der Länder, Einrichtungen der Daseinsvorsorge zu unterstützen, sofern die Mittel der Kommunen bzw. anderer Träger nicht ausreichen. Mit der vorgeschlagenen neuen GRD sollen sich Bund und Länder mit je $50 \%$ der Kosten an einem bundesweiten Förderprogramm beteiligen. Das Programm soll konkretisieren, welche Arten von Einrichtungen sowie welche Regionen aufgrund welcher Indika-

(C) Der/die Autor:in(nen) 2021. Open Access: Dieser Artikel wird unter der Creative Commons Namensnennung 4.0 International Lizenz veröffentlicht (creativecommons.org/licenses/by/4.0/deed.de).

Open Access wird durch die ZBW - Leibniz-Informationszentrum Wirtschaft gefördert. toren Fördermittel erhalten und welche Versorgungsstandards erreicht werden sollen.

Hierzu ist zunächst zu sagen, dass es aufgrund finanzieller Restriktionen sowie Wirtschaftlichkeitsüberlegungen bezüglich der erforderlichen Auslastung von Einrichtungen der Daseinsvorsorge realistischerweise weder mit einer GRD noch mit anderen Maßnahmen gelingen kann, für jeden Ort jede Art von Daseinsvorsorge zu gewährleisten. Gegen eine GRD sprechen die bekannten Nachteile der Mischfinanzierung sowie der Lenkung kommunaler Entscheidungen über den „goldenen Zügel“ der Vergabe von Fördermitteln: Auf jeder der beteiligten Ebenen müssen Fachkräfte für die Planung und Abwicklung der Förderpolitik vorgehalten werden (Mehrfach-Bürokratie). Auch bei den Empfangenden entsteht ein hoher Verwaltungsaufwand. Da zumeist Investitionen gefördert werden, besteht die Gefahr, dass die Kommunen auf Folgekosten sitzen bleiben. Durch die neue Gemeinschaftsaufgabe wäre eine weitere Ausbreitung der Fördermentalität zu erwarten - die Einwerbung von Mitteln wird als Erfolg verbucht, die tatsächlichen Bedarfe treten in den Hintergrund.

Die Forderung nach einer GRD dürfte auch daraus resultieren, dass die Politik zumeist Förderprogramme - bei denen es scheinbar nur Gewinnende gibt - gegenüber Veränderungen auf der Einnahmenseite - durch die es immer auch Verlierende gibt - bevorzugt. Es wäre aber besser, die allgemeine finanzielle Ausstattung der Kommunen in strukturschwachen Regionen so zu gestalten, dass die Kommunen und die dort lebenden Menschen wieder selbst entscheiden können, welche Einrichtungen der Daseinsvorsorge erhalten bzw. erweitert werden sollen. Die Politik sollte deshalb ihre Bemühungen darauf richten, endlich eine grundlegende Reform der kommunalen Steuereinnahmen in die Wege zu leiten, die zu einem räumlich gleichmäßigeren Steueraufkommen führt. Hierfür gibt es diverse Vorschläge, von denen nahezu jeder besser ist als der Status quo mit seinen (vor allem durch die heutige Form der Gewerbesteuer bedingten) Verteilungswirkungen zuungunsten strukturschwacher Räume. Zudem sollten kooperative Ansätze im Bereich der Daseinsvorsorge verstärkt Beachtung finden, durch die sich der kommunale Finanzbedarf reduzieren und die Versorgung verbessern lässt. Hier sind die interkommunale Zusammenarbeit sowie das Instrument der lokalen (Infrastruktur-)Genossenschaften als Träger der Daseinsvorsorge angesprochen. Genossenschaftliche Arrangements haben zudem den Vorteil, auch das bürgerschaftliche Engagement und die Identifikation der Menschen mit inrer Kommune oder Region zu stärken.

Martin T.W. Rosenfeld Martin-Luther-Universität Halle-Wittenberg martin.rosenfeld@wiwi.uni-halle.de 